\title{
ÓXIDOS DO TIPO PEROVSKITA PARA REAÇÃO DE REDUÇÃO DE NO COM CO
}

\author{
Eurico Y. Tanabe e Elisabete M. Assaf *
}

Instituto de Química de São Carlos, Universidade de São Paulo, CP 780, 13560-970 São Carlos - SP, Brasil

Recebido em 3/4/08; aceito em 9/12/08; publicado na web em 28/5/09

\begin{abstract}
PEROVSKITE-TYPE OXIDES TO NO REDUCTION WITH CO. In this work, the perovskite-type oxides $\mathrm{LaNiO}_{3}, \mathrm{LaMnO}_{3}$, $\mathrm{La}_{0,7} \mathrm{Sr}_{0,3} \mathrm{NiO}_{3}$ and $\mathrm{La}_{0,7} \mathrm{Sr}_{0,3} \mathrm{MnO}_{3}$ were prepared by co-precipitation and tested in the NO reduction with $\mathrm{CO}$ at 400 and $500{ }^{\circ} \mathrm{C}$ for $10 \mathrm{~h}$. The catalysts were characterized by X-ray diffraction, temperature programmed reduction with hydrogen, nitrogen adsorption and chemical analysis. The nonstoichiometric oxygen was quantified by temperature programmed reduction, and the catalytic tests showed that the $\mathrm{La}_{0,7} \mathrm{Sr}_{0,3} \mathrm{MnO}_{3}$ catalyst presented the higher performance for the reduction reaction of NO with CO. The partial substitution of lanthanum by strontium increased the NO conversion and the $\mathrm{N}_{2}$ yield.
\end{abstract}

Keywords: perovskite; lanthanum; NO reduction with CO.

\section{INTRODUÇÃO}

Mundialmente, os problemas ambientais, como a poluição atmosférica resultante da emissão de gases lançados na atmosfera, estão sendo discutidos e debatidos através de conferências e encontros de governantes de vários países, a fim de criar uma rigorosa política ambiental para controlar e limitar a quantidades de gases emitidos. Gases como NO, $\mathrm{CO}, \mathrm{C}_{\mathrm{x}} \mathrm{H}_{\mathrm{y}}$, e $\mathrm{SO}_{\mathrm{x}}$, além de contribuírem para o efeito estufa, possuem poder de destruição da camada de ozônio. Um dos grandes marcos na tentativa de minimizar a quantidade de gases lançados na atmosfera foi a obrigatoriedade do uso de catalisador (contendo metais nobres, como paládio, platina e rutênio) nos veículos automotivos, convertendo os gases da combustão incompleta em $\mathrm{CO}_{2}, \mathrm{H}_{2} \mathrm{O}$ e $\mathrm{N}_{2}$.

Nos últimos anos, os óxidos do tipo Perovskita (fórmula geral $\mathrm{ABO}_{3}$ ) têm sido reconhecidos como catalisadores ativos, em substituição aos metais nobres, para uma variedade de reações, especialmente em catálise ambiental, como tratamento dos gases de exaustão, combustão catalítica de hidrocarbonetos, oxidação de $\mathrm{CO}$ e hidrocarbonetos, redução de $\mathrm{NO}$ com $\mathrm{CO}$, reações de reforma e outras. $^{1-5}$

A formação dos óxidos do tipo perovskita só ocorre quando a relação entre os raios dos íons metálicos envolvidos obedece ao fator de tolerância $t$, que deve estar entre $0,75-1,0\left(t=\left(r_{A}+r_{O}\right) / 2^{1 / 2}\left(r_{B}+\right.\right.$ $\left.\mathrm{r}_{\mathrm{o}}\right)$ ). Uma das grandes propriedades destes óxidos é a possibilidade de substituição parcial dos cátions $\mathrm{A}$ e/ou $\mathrm{B}$, levando a uma grande classe de materiais de fórmula geral $\mathrm{A}_{1-\mathrm{x}} \mathrm{A}_{\mathrm{x}}^{\prime} \mathrm{B}_{1-\mathrm{y}} \mathrm{B}_{\mathrm{y}}^{\prime} \mathrm{O}_{3+\delta}$. Na fórmula, $\delta$ representa o excesso ou deficiência de oxigênio devido à não estequiometria destas espécies. A substituição parcial de A e/ou B por metais com diferentes estados de oxidação gera defeitos na estrutura (vacâncias aniônicas ou catiônicas), que são geralmente associados a propriedades físicas e químicas do material, favorecendo, por exemplo, o transporte de íons dentro da estrutura, provocando interessantes diferenças no desempenho catalítico. ${ }^{6}$

Considerando os problemas causados pela poluição automotiva e a necessidade de encontrar catalisadores ativos e estáveis que possam substituir os metais nobres presentes no conversor catalítico automotivo, o objetivo do presente trabalho foi preparar, caracterizar e estudar o comportamento dos catalisadores do tipo perovskita baseados em lantânio, especificamente $\mathrm{LaNiO}_{3}, \mathrm{LaMnO}_{3}, \mathrm{La}_{0,7} \mathrm{Sr}_{0,3} \mathrm{NiO}_{3} \mathrm{e}$ $\mathrm{La}_{0,7} \mathrm{Sr}_{0,3} \mathrm{MnO}_{3}$, frente à reação de redução do NO com CO.

*e-mail: eassaf@iqsc.usp.br

\section{PARTE EXPERIMENTAL}

\section{Preparação dos catalisadores}

Os óxidos do tipo perovskitas $\mathrm{LaNiO}_{3}, \mathrm{LaMnO}_{3}, \mathrm{La}_{0,7} \mathrm{Sr}_{0,3} \mathrm{NiO}_{3}$ e $\mathrm{La}_{0,7} \mathrm{Sr}_{0,3} \mathrm{MnO}_{3}$ foram preparados baseando-se no método da coprecipitação descrito por Liu et al.. ${ }^{7}$ Inicialmente foram preparadas duas soluções: (A) solução de $\mathrm{Na}_{2} \mathrm{CO}_{3} / \mathrm{NaOH} ;\left[\mathrm{Na}^{+}\right]=3,0 \mathrm{~mol} \mathrm{~L}^{-1} \mathrm{e}$ (B) solução dos nitratos metálicos [metal] $=1,0 \mathrm{~mol} \mathrm{~L}^{-1}$. Em seguida, simultaneamente e gota a gota, as duas soluções foram adicionadas em um béquer contendo $100 \mathrm{~mL}$ de água destilada. Durante essa adição, o pH da solução foi mantido constante e igual a 10. Após a adição da solução dos nitratos metálicos, formou-se um precipitado gelatinoso que foi lavado com fluxo de água destilada $\left(2 \mathrm{~L} \mathrm{~h}^{-1}\right)$ durante $5 \mathrm{~h} \mathrm{e}$, em seguida, com etanol $(150 \mathrm{~mL})$ no intervalo de $1 \mathrm{~h}$. O precipitado foi então seco em estufa $\left(90^{\circ} \mathrm{C}\right)$ durante $3 \mathrm{~h}$, pré-calcinado a 350 ${ }^{\circ} \mathrm{C}$ durante $2 \mathrm{~h}$ e calcinado a $800{ }^{\circ} \mathrm{C}$ durante $4 \mathrm{~h}$, sob fluxo de ar de $15 \mathrm{~mL} \mathrm{~min}{ }^{-1}$ e rampa de aquecimento de $5{ }^{\circ} \mathrm{C} \mathrm{min}^{-1}$, para se obter o óxido do tipo perovskita.

\section{Caracterização dos catalisadores}

Os catalisadores foram caracterizados por fisissorção de nitrogênio, difração de raios-X (DRX), redução à temperatura programada com hidrogênio $\left(\mathrm{RTP}-\mathrm{H}_{2}\right)$ e espectrometria de emissão por plasma induzido (ICP). As análises de DRX foram realizadas em um equipamento Rigaku-Miniflex, operado com uma radiação $\mathrm{CuK} \alpha(\lambda=$ $1,5418)$ entre 3 e $80^{\circ}(2 \theta)$ e velocidade do goniômetro de $0,5 \%$ min. Nas análises de RTP- $\mathrm{H}_{2}$, em um equipamento Micromeritcs Chemissorb 2705 , foram utilizados $10 \mathrm{mg}$ de amostra, fluxo de $30 \mathrm{~mL} \mathrm{~min}^{-1} \mathrm{de}$ $5 \% \mathrm{H}_{2} / \mathrm{N}_{2}$ e taxa de aquecimento de $10^{\circ} \mathrm{C} \mathrm{min}^{-1}$. A área superficial dos catalisadores foi determinada por fisissorção de $\mathrm{N}_{2}$ a $77 \mathrm{~K}$, utilizando a equação de B.E.T em um equipamento Quantachrome Nova 2.0. Os teores dos metais nos catalisadores foram determinados através de ICP em um espectrômetro Perkin Elmer Optima-3000.

\section{Avaliação catalítica}

Os catalisadores, óxidos do tipo perovskitas, foram avaliados na reação de redução de NO com CO nas temperaturas de 400 e $500{ }^{\circ} \mathrm{C}$. Os testes catalíticos foram realizados em um reator tubular de vidro com leito fixo, utilizando $150 \mathrm{mg}$ de catalisador. Durante a reação, 
o reator foi alimentado com $60 \mathrm{~mL} \mathrm{~min}{ }^{-1}$ de uma mistura (1:1) de CO e NO. Ambos os gases são diluídos em hélio (1\% CO:99\% He) e (1\% NO:99\% He). Os produtos da reação foram analisados em um cromatógrafo a gás com detector de condutividade térmica, utilizando duas colunas empacotadas em série, uma coluna Porapak N (2 m x 1/8 "), uma peneira molecular 13X (3 m x 1/8") e hélio como gás de arraste. A quantificação dos gases foi realizada baseando-se no método do padrão externo.

\section{RESULTADOS E DISCUSSÃO}

A Tabela 1 apresenta os resultados da área superficial específica dos catalisadores preparados pelo método da co-precipitação e os resultados das áreas superficiais obtidas por outros autores, utilizandose diferentes métodos..$^{7-9}$

As áreas superficiais obtidas a partir do método da co-precipitação foram maiores que através dos outros métodos, demonstrando assim sua eficácia. A adição parcial do estrôncio provocou um aumento no valor das áreas superficiais, para ambos os catalisadores. Esse aumento foi observado também por Liu et al.. ${ }^{7}$ Este fato pode estar associado ao maior raio iônico do estrôncio $\left(\mathrm{Sr}^{2+}=0,113 \mathrm{~nm}\right)$, quando comparado ao do lantânio $\left(\mathrm{La}^{3+}=0,106 \mathrm{~nm}\right)$. De acordo com a literatura, ${ }^{2}$ a adição ou substituição de um átomo por outro pode acarretar um desbalanço eletrônico em função da carga e, também, modificação na estrutura devido à diferença do raio atômico, influenciando no tamanho da partícula e no valor da sua área superficial específica.

Tabela 1. Área superficial dos catalisadores

\begin{tabular}{lcc}
\hline Catalisador & \multicolumn{2}{c}{$\begin{array}{c}\text { Área superficial }\left(\mathrm{m}^{2} \mathrm{~g}^{-1}\right) \\
\text { Co-precipitado }\end{array}$} \\
\hline $\mathrm{LaNiO}_{3}$ & 7 & Outros métodos \\
$\mathrm{La}_{0,7} \mathrm{Sr}_{0,3} \mathrm{NiO}_{3}$ & 10 & $1,38^{(\text {a) }}$ \\
$\mathrm{LaMnO}_{3}$ & 13 & ----- \\
$\mathrm{La}_{0,7} \mathrm{Sr}_{0,3} \mathrm{MnO}_{3}$ & 15 & $8,79^{(\text {b) }}$ \\
\hline
\end{tabular}

(a) Citrato (8); (b) decomposição por spray (9); (c) cerâmico (10).

Na Figura 1, são apresentados os resultados de DRX dos catalisadores. Todos, com exceção do $\mathrm{La}_{0,7} \mathrm{Sr}_{0,3} \mathrm{NiO}_{3}$ estão de acordo com o padrão de difração da estrutura do óxido do tipo perovskita, obtido na literatura. ${ }^{10}$ Observa-se que os difratogramas referentes aos catalisadores $\mathrm{LaMnO}_{3}$ (Figura 1a) e $\mathrm{La}_{0,7} \mathrm{Sr}_{03} \mathrm{MnO}_{3}$ (Figura 1b) são muito semelhantes e apresentam os principais picos em $2 \theta=22,5^{\circ}$; $32,1^{\circ} ; 39,1^{\circ} ; 46,1^{\circ} ; 52,0^{\circ} ; 57,5^{\circ} ; 67,7^{\circ}$ e $78,1^{\circ}$. Segundo o JCPDS,,$^{10}$ a diferença entre os difratogramas do $\mathrm{LaMnO}_{3}$ e $\mathrm{La}_{0,7} \mathrm{Sr}_{0,3} \mathrm{MnO}_{3}$ está na intensidade dos picos em $2 \theta=22,5^{\circ}$ e $39,1^{\circ}$. Para o catalisador $\mathrm{LaMnO}_{3}$ o sinal em $22,5^{\circ}$ é mais intenso que o sinal em $39,1^{\circ}$, enquanto que para o $\mathrm{La}_{0,7} \mathrm{Sr}_{0,3} \mathrm{MnO}_{3}$ é ao contrário, ou seja o sinal em $22,5^{\circ}$ é menos intenso que em $39,1^{\circ}$. Esta diferença de intensidade entre os sinais também foi observada neste trabalho.

No difratograma do $\mathrm{LaNiO}_{3}$ (Figura 1c), os picos de maior intensidade localizam-se em $2 \theta=32,9^{\circ} ; 47,4^{\circ} ; 58,8^{\circ} ; 23,3^{\circ} ; 40,8^{\circ} 41,3^{\circ} ; 67,4^{\circ}$ e $79,2^{\circ}$ e estão de acordo com a literatura. ${ }^{10}$ Os sinais em $2 \theta=37,3^{\circ}$; $43,3^{\circ}$ e 62,9 podem ser atribuídos à fase NiO. Segundo Dupeyrat et al., ${ }^{11}$ pequenos cristais de $\mathrm{NiO}$ agregados podem ser formados juntamente com a estrutura do óxido do tipo perovksita $\mathrm{LaNiO}_{3}$. Para o catalisador $\mathrm{La}_{0,7} \mathrm{Sr}_{0,3} \mathrm{NiO}_{3}$ (Figura 1d), além dos picos referentes à estrutura da perovskita $\mathrm{LaNiO}_{3}$, observam-se picos referentes aos óxidos de níquel (NiO) e lantânio $\left(\mathrm{La}_{2} \mathrm{O}_{3}\right)$. Picos referentes ao óxido de estrôncio não foram observados, possivelmente devido à baixa concentração do metal, ou à incorporação do estrôncio na estrutura.
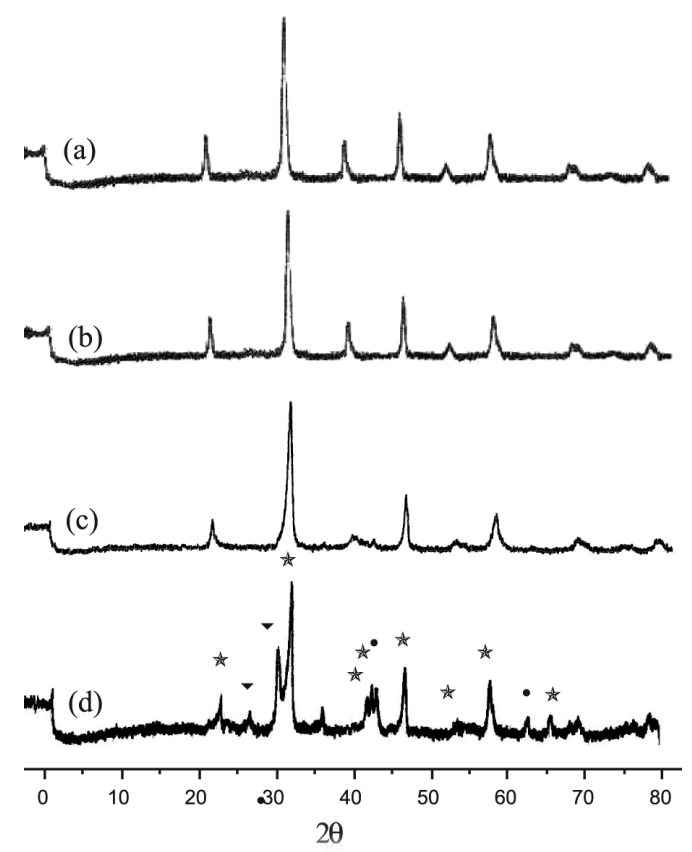

Figura 1. Difratogramas de raios-X dos catalisadores: (a)LaMnO, (b) $\mathrm{La}_{0.7} \mathrm{Sr}_{0,3} \mathrm{MnO}_{3},(\mathrm{c}) \mathrm{LaNiO}_{3},(d) \mathrm{La}_{0,7} \mathrm{Sr}_{0,3} \mathrm{NiO}_{3} \cdot \bullet \mathrm{NiO} \vee \mathrm{La}_{2} \mathrm{O}_{3} \star \mathrm{LaNiO}_{3}$

A Tabela 2 mostra os resultados de análise química e observase que a quantidade real dos metais contida nos catalisadores está próxima aos valores teóricos.

Os perfis de RTP- $\mathrm{H}_{2}$ dos catalisadores $\mathrm{LaNiO}_{3}, \mathrm{La}_{0,7} \mathrm{Sr}_{0,3} \mathrm{NiO}_{3}$, $\mathrm{LaMnO}_{3}$ e $\mathrm{La}_{07} \mathrm{Sr}_{03} \mathrm{MnO}_{3}$ são mostrados nas Figuras 2 e 3. De acordo com Wang et al. ${ }^{12}$ e Lee et al., ${ }^{13}$ a redução do estrôncio não pode ser observada dentro dessa faixa de temperatura. O perfil de RTP do manganês presente no óxido do tipo perovskita $\mathrm{LaMnO}_{3}$ (Figura 2, curva a) apresenta dois picos de redução, porém para o intervalo de temperatura analisada $\left(<1000{ }^{\circ} \mathrm{C}\right)$ não é possível a redução do $\mathrm{Mn}^{2+}$ para $\mathrm{Mn}^{0}$. De acordo com a literatura, ${ }^{12-15}$ normalmente, o óxido do tipo perovskita $\mathrm{LaMnO}_{3}$ apresenta-se como $\mathrm{LaMnO}_{3+\delta}$ devido ao excesso de oxigênio não estequiométrico contido na sua estrutura, acarretado pelo aumento no estado de oxidação do $\mathrm{Mn}^{3+}$ para $\mathrm{Mn}^{4+}$. Alguns pesquisadores ${ }^{15-17}$ calcularam o teor de $\mathrm{Mn}^{4+}$ nas perovskitas $\mathrm{LaMnO}_{3+\delta}$, que pode atingir em média até $35 \%$. Rojas et al. ${ }^{15}$ realizaram estudos com $\mathrm{LaMnO}_{3+\delta}$ e verificaram que o manganês pode perder mais que 1 elétron por molécula reduzida.

Tabela 2. Resultado da análise elementar por ICP

\begin{tabular}{lccc}
\hline \multicolumn{2}{l}{ Catalisador / Elementos } & $\begin{array}{c}\text { Concentração } \\
\text { teórica (ppm) }\end{array}$ & $\begin{array}{c}\text { Concentração real } \\
\text { (ppm) }\end{array}$ \\
\hline $\mathrm{LaNiO}_{3}$ & $\mathrm{La}$ & 8,96 & 8,84 \\
$\mathrm{LaMnO}_{3}$ & $\mathrm{Ni}$ & 3,82 & 3,53 \\
& $\mathrm{La}$ & 8,04 & 8,09 \\
$\mathrm{La}_{0,7} \mathrm{Sr}_{0,3} \mathrm{NiO}_{3}$ & $\mathrm{Mn}$ & 3,19 & 2,64 \\
& $\mathrm{Sr}$ & 7,60 & 7,55 \\
& $\mathrm{Ni}$ & 4,01 & 1,90 \\
& $\mathrm{La}$ & 8,58 & 4,58 \\
& $\mathrm{Sr}$ & 2,32 & 8,54 \\
$\mathrm{La}_{0,7} \mathrm{Sr}_{0,3} \mathrm{MnO}_{3}$ & $\mathrm{Mn}$ & 4,86 & 2,21 \\
& & & 4,58 \\
\hline
\end{tabular}


Na Figura 2, curva a, RTP do $\mathrm{LaMnO}_{3+\delta}$, o pico de redução em $516{ }^{\circ} \mathrm{C}$ pode ser atribuído à redução do $\mathrm{Mn}^{4+}$ para $\mathrm{Mn}^{3+}$, incluindo o excesso de oxigênio (Equação 1) e o pico em $846^{\circ} \mathrm{C}$ à redução do $\mathrm{Mn}^{3+}$ para $\mathrm{Mn}^{2+}$ (Equação 2). Esses mesmos picos, juntamente com ombros, podem ser observados quando se adiciona parcialmente o estrôncio (Figura 2, curva b). A substituição parcial do lantânio pelo estrôncio provoca um desbalanceamento de cargas e, para compensá-lo, há um aumento na quantidade da espécie $\mathrm{Mn}^{4+}$, através da oxidação do $\mathrm{Mn}^{3+}$ a $\mathrm{Mn}^{4+}$, ou uma deficiência de oxigênio na estrutura.

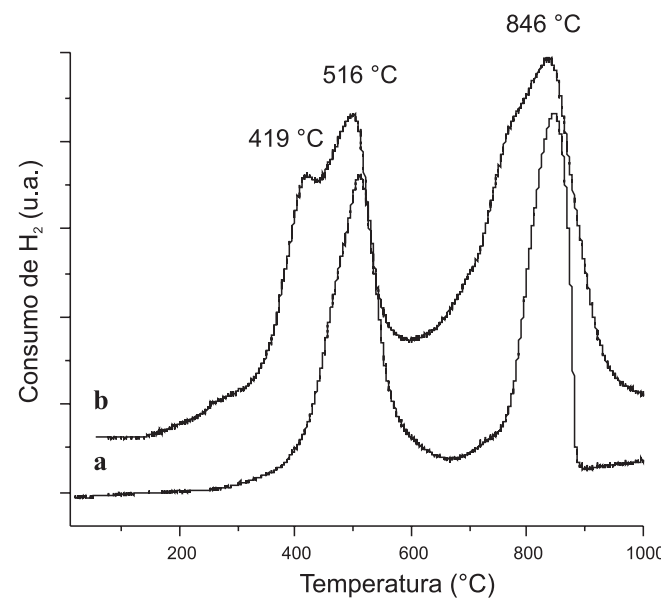

Figura 2. Perfis de redução à temperatura programada para os catalisadores: (a) $\mathrm{LaMnO}_{3}$ e (b) $\mathrm{La}_{0,7} \mathrm{Sr}_{0,3} \mathrm{MnO}_{3}$

$\mathrm{LaMnO}_{3+\delta}+\delta \mathrm{H}_{2} \rightarrow \mathrm{LaMnO}_{3}+\delta \mathrm{H}_{2} \mathrm{O}$

$2 \mathrm{LaMnO}_{3}+\mathrm{H}_{2} \rightarrow \mathrm{La}_{2} \mathrm{O}_{3}+2 \mathrm{MnO}+\mathrm{H}_{2} \mathrm{O}$

Observando-se o perfil de RTP para o catalisador $\mathrm{LaNiO}_{3}(\mathrm{Fi}-$ gura 3 , curva a), verifica-se que apresenta três picos de redução. O pico em $435{ }^{\circ} \mathrm{C}$ refere-se à redução do $\mathrm{Ni}^{3+}$ para $\mathrm{Ni}^{2+}$, resultando em $\mathrm{La}_{2} \mathrm{Ni}_{2} \mathrm{O}_{5}$ (Equação 3) e este se reduz de $\mathrm{Ni}^{2+}$ para $\mathrm{Ni}^{0}$ a 580 ${ }^{\circ} \mathrm{C}$ (Equação 4). O pico em $420{ }^{\circ} \mathrm{C}$ pode ser atribuído a redução da fase $\mathrm{NiO}$ segregada (Equação 5), corroborando com os resultados já apresentados de DRX.

$$
\begin{aligned}
& 2 \mathrm{LaNiO}_{3}+\mathrm{H}_{2} \rightarrow \mathrm{La}_{2} \mathrm{Ni}_{2} \mathrm{O}_{5}+\mathrm{H}_{2} \mathrm{O} \\
& \mathrm{La}_{2} \mathrm{Ni}_{2} \mathrm{O}_{5}+\mathrm{H}_{2} \rightarrow \mathrm{La}_{2} \mathrm{O}_{3}+2 \mathrm{Ni}^{0}+2 \mathrm{H}_{2} \mathrm{O} \\
& \mathrm{NiO}+\mathrm{H}_{2} \rightarrow \mathrm{Ni}^{0}+\mathrm{H}_{2} \mathrm{O}
\end{aligned}
$$

A adição parcial do estrôncio na estrutura do $\mathrm{LaNiO}_{3}$ especificamente $\mathrm{La}_{0,7} \mathrm{Sr}_{0,3} \mathrm{NiO}_{3}$ (Figura 3, curva b), ocasionou o aparecimento de novos picos entre as temperaturas 713 a $780^{\circ} \mathrm{C}$. Entretanto, o primeiro pico, em $357^{\circ} \mathrm{C}$, pode ser atribuído à redução do $\mathrm{NiO}$ presente juntamente com a fase perovskita, pois no DRX verificou-se a presença do óxido de níquel. Em $418^{\circ} \mathrm{C}$, pode ser atribuído à redução do $\mathrm{Ni}^{3+}$ para $\mathrm{Ni}^{2+} \mathrm{e}$, em $608{ }^{\circ} \mathrm{C}$, à redução do $\mathrm{Ni}^{2+}$ para $\mathrm{Ni}^{\circ}$.

A partir do consumo total de hidrogênio, obtido através das áreas sob as curvas dos perfis de RTP, foi determinada a quantidade total de oxigênio removida por mol de óxido do tipo perovskita ${ }^{18} \mathrm{e}$ o excesso ou a deficiência de oxigênio para cada amostra $(\delta)$ foi calculado através da metodologia baseada no trabalho de Buciuman et al.. ${ }^{19} \mathrm{Os}$ consumos teóricos de hidrogênio, juntamente com os obtidos neste trabalho, representados pelas áreas sob as curvas, estão apresentados na Tabela 3. Para os catalisadores $\mathrm{LaMnO}_{3} \mathrm{e} \mathrm{La}_{0,7} \mathrm{Sr}_{0,3} \mathrm{MnO}_{3}$ as áreas do RTP são maiores que as teóricas, indicando que estes óxidos apresentam excesso de oxigênio na sua estrutura. Já os catalisadores $\mathrm{LaNiO}_{3} \mathrm{e} \mathrm{La}_{0,7} \mathrm{Sr}_{0,3} \mathrm{NiO}_{3}$ apresentaram áreas menores que as teóricas, sugerindo deficiência de oxigênio na estrutura.

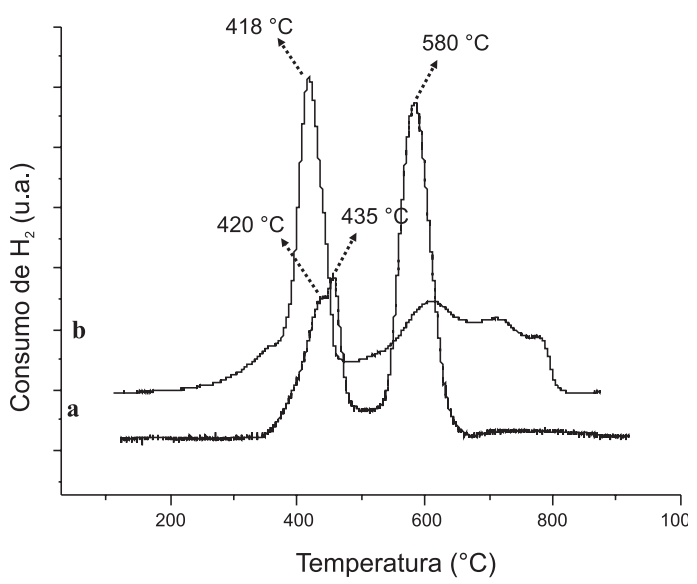

Figura 3. Perfis de redução à temperatura programada para os catalisadores: (a) $\mathrm{LaNiO}_{3}$ e (b) $\mathrm{La}_{0,3} \mathrm{Sr}_{0,3} \mathrm{NiO}_{3}$

Tabela 3. Dados de RTP

\begin{tabular}{lcc}
\hline Catalisador & Área teórica (u.a.) & Área RTP (u.a.) \\
\hline $\mathrm{LaMnO}_{3}$ & $48,5 \times 10^{3}$ & $74,2 \times 10^{3}$ \\
$\mathrm{La}_{0,7} \mathrm{Sr}_{0,3} \mathrm{MnO}_{3}$ & $65,6 \times 10^{3}$ & $68,7 \times 10^{3}$ \\
$\mathrm{LaNiO}_{3}$ & $142,1 \times 10^{3}$ & $139,6 \times 10^{3}$ \\
$\mathrm{La}_{0,7} \mathrm{Sr}_{0,3} \mathrm{NiO}_{3}$ & $167,8 \times 10^{3}$ & $133,1 \times 10^{3}$ \\
\hline
\end{tabular}

massa da amostra (RTP): $10 \mathrm{mg}$

Os valores calculados do excesso e/ou deficiência de oxigênio

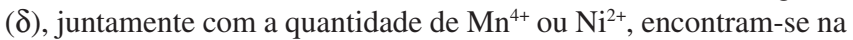
Tabela 4.

Tabela 4. Quantidade de $\mathrm{Mn}^{4+}$ e $\mathrm{Ni}^{2+}$ e valores de $\delta$

\begin{tabular}{lcc}
\hline Catalisador & \multicolumn{1}{c}{ Espécies } & $\delta$ \\
\hline $\mathrm{LaMnO}_{3+\delta}$ & $52 \% \mathrm{Mn}^{4+} /\left(\mathrm{Mn}^{4+}+\mathrm{Mn}^{3+}\right)$ & 0,26 \\
$\mathrm{La}_{0,7} \mathrm{Sr}_{0,3} \mathrm{MnO}_{3+\delta}$ & $42 \% \mathrm{Mn}^{4+} /\left(\mathrm{Mn}^{4+}+\mathrm{Mn}^{3+}\right)$ & 0,06 \\
$\mathrm{LaNiO}_{3-\delta}$ & $8 \% \mathrm{Ni}^{2+} /\left(\mathrm{Ni}^{3+}+\mathrm{Ni}^{2+}\right)$ & 0,04 \\
$\mathrm{La}_{0,7} \mathrm{Sr}_{0,3} \mathrm{NiO}_{3-\delta}$ & $32 \% \mathrm{Ni}^{2+} /\left(\mathrm{Ni}^{3+}+\mathrm{Ni}^{2+}\right)$ & 0,16 \\
\hline
\end{tabular}

\section{Avaliação catalítica}

Os resultados das reações de redução de NO com CO, a 400 e $500{ }^{\circ} \mathrm{C}$, são apresentados nas Figuras 4-7.

Comparando-se os catalisadores $\mathrm{LaNiO}_{3}$ (Figura 4) e $\mathrm{LaMnO}_{3}$ (Figura 5), o catalisador $\mathrm{LaNiO}_{3}$ foi o que apresentou o melhor resultado em relação à conversão dos reagentes, tanto a 400 quanto a $500{ }^{\circ} \mathrm{C}$. Porém, quando o lantânio foi substituído parcialmente pelo estrôncio houve uma melhora significativa na atividade e estabilidade dos catalisadores e o $\mathrm{La}_{0,7} \mathrm{Sr}_{0,3} \mathrm{MnO}_{3}$ mostrou conversão superior à obtida com o $\mathrm{La}_{0,7} \mathrm{Sr}_{0,3} \mathrm{NiO}_{3}$.

A conversão do NO foi, para quase todos os catalisadores e temperaturas, com exceção do $\mathrm{LaNiO}_{3}$, superior à do $\mathrm{CO}$. Esta maior conversão pode ser atribuída à ocorrência simultânea das reações de redução de NO com CO e de decomposição direta de NO. Esta hipótese foi baseada no cálculo do balanço de massa, considerando-se a 


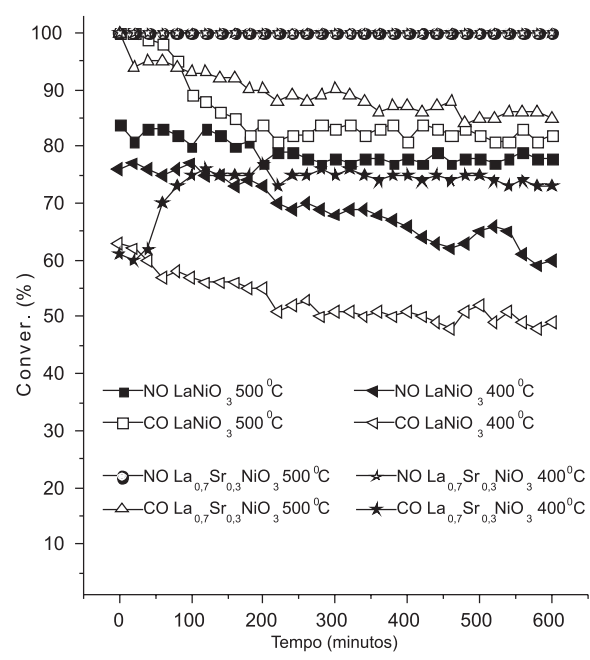

Figura 4. Conversão do NO e CO a 400 e $500^{\circ} \mathrm{C}$ em função do tempo para catalisador contendo níquel

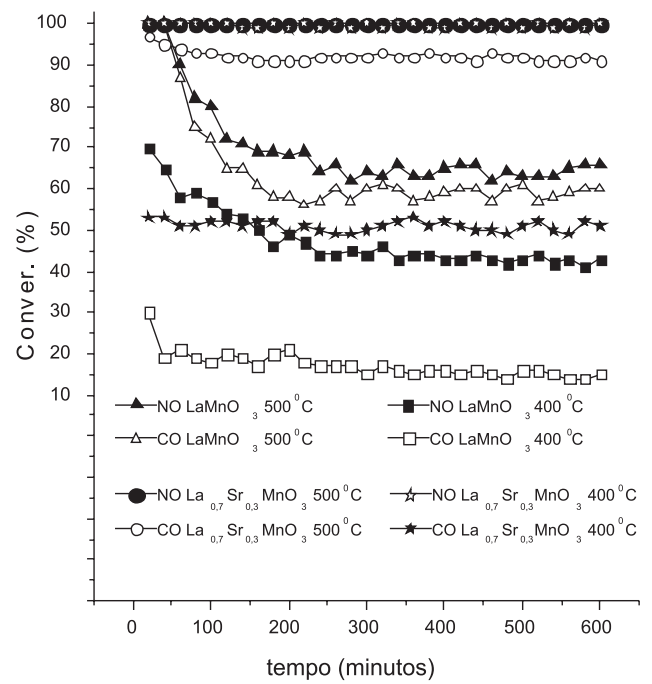

Figura 5. Conversão do NO e CO a 400 e $500{ }^{\circ} \mathrm{C}$ em função do tempo para catalisador contendo manganês

estequiometria das reações. Para o catalisador $\mathrm{LaNiO}_{3}$, a seletividade para a reação de oxidação do CO parece ser ligeiramente superior.

As medidas de atividade catalítica mostraram que, ao se substituir parcialmente o lantânio pelo estrôncio, houve uma melhora significativa na reação de redução de NO com CO. Dentre os catalisadores não substituídos, o que apresentou melhor atividade catalítica foi o $\mathrm{LaNiO}_{3-\delta}$, sendo que este catalisador se apresentou como óxido deficiente em oxigênio, enquanto que o catalisador que apresentou uma menor atividade catalítica, $\mathrm{LaMnO}_{3+\delta}$, se apresentou como óxido com excesso de oxigênio. $\mathrm{O}$ mesmo fato ocorreu quando os catalisadores foram substituídos parcialmente pelo estrôncio, ou seja, as melhores atividades foram obtidas para os catalisadores que se apresentaram deficientes em oxigênio.

As Figuras 6 e 7 mostram o rendimento em $\mathrm{N}_{2}$ obtido para os catalisadores e temperaturas estudadas. Pode-se observar que as maiores temperaturas de reação e a substituição do lantânio pelo estrôncio favoreceram a formação de nitrogênio.

Em todos os ensaios catalíticos não foi observada a presença de $\mathrm{N}_{2} \mathrm{O}$ e os catalisadores demonstraram ser estáveis após as primeiras horas de reação.

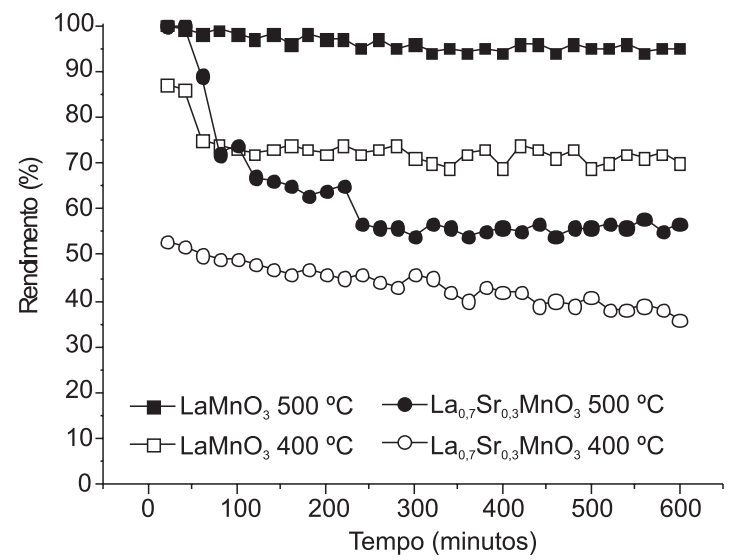

Figura 6. Rendimento em $\mathrm{N}_{2}$ a 400 e $500^{\circ} \mathrm{C}$ em função do tempo para catalisadores contendo manganês

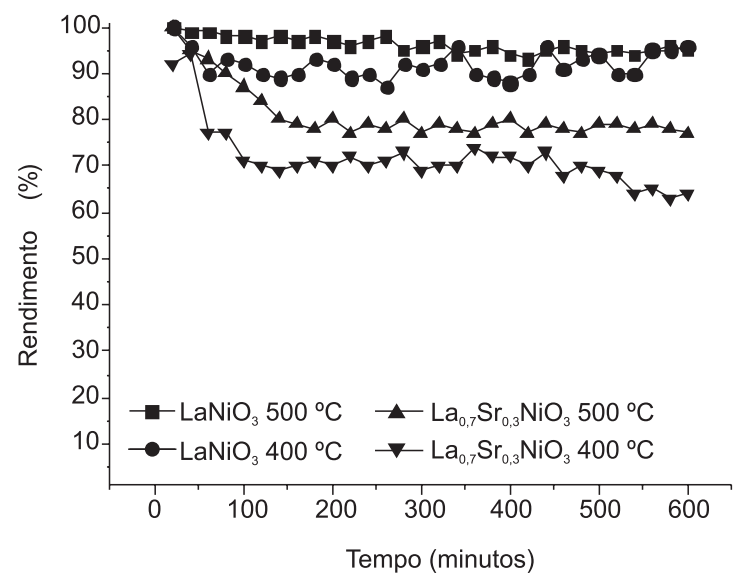

Figura 7. Rendimento em $\mathrm{N}_{2}$ a 400 e $500^{\circ} \mathrm{C}$ em função do tempo para catalisadores contendo níquel

Para a reação estudada é possível sugerir o seguinte mecanismo reacional: primeiramente a molécula de $\mathrm{NO}$ é adsorvida sobre o catalisador e reduzida a $\mathrm{N}_{2}$, deixando o catalisador oxidado. A presença do CO facilmente reduz o metal novamente ao seu estado inicial, dessorvendo-se sob a forma de $\mathrm{CO}_{2}$. Assim, a etapa limitante no mecanismo é a redução do NO e oxidação do catalisador. De acordo com este mecanismo, os óxidos do tipo perovskita que possuem deficiência em oxigênio serão mais ativos para a reação de redução do NO com $\mathrm{CO}$, devido ao menor estado de oxidação em que se encontra o metal ativo do catalisador.

\section{CONCLUSÕES}

O método da co-precipitação mostrou-se eficiente para a obtenção dos óxidos do tipo perovskita com áreas específicas variando entre 7 e $15 \mathrm{~m}^{2} / \mathrm{g}$.

De acordo com os dados de RTP, foi possível verificar a não estequiometria dos óxidos do tipo perovskita, através da quantificação da deficiência ou do excesso de oxigênio contido nas amostras. Assim, os óxidos contendo níquel foram classificados como deficientes em oxigênio, $\mathrm{LaNiO}_{2,96} \mathrm{e} \mathrm{La}_{0,7} \mathrm{Sr}_{0,3} \mathrm{NiO}_{2,84}$, enquanto que os óxidos contendo manganês como excesso em oxigênio, $\mathrm{LaMnO}_{3,26}$ e $\mathrm{La}_{0,7} \mathrm{Sr}_{0,3} \mathrm{MnO}_{3,06}$.

A adição ou substituição de outros metais na estrutura do óxido tipo perovskita acarreta a existência de oxigênio não estequiométrico, que melhora a atividade catalítica, o que foi comprovado com a adição de estrôncio nos catalisadores $\mathrm{LaNiO}_{3}$ e $\mathrm{LaMnO}_{3}$. Dentre os 
catalisadores estudados, os que apresentaram melhor atividade foram aqueles com maior deficiência em oxigênio. A atividade mostrou-se decrescente para os catalisadores na seguinte ordem: $\mathrm{La}_{0,7} \mathrm{Sr}_{0,3} \mathrm{NiO}_{2,84}$ $>\mathrm{LaNiO}_{2,96}>\mathrm{La}_{0,7} \mathrm{Sr}_{0,3} \mathrm{MnO}_{3,06}>\mathrm{LaMnO}_{3,26}$.

A maior atividade catalítica mostrada pelos óxidos do tipo perovskita contendo níquel indica que estes catalisadores são promissores para a reação de oxidação de NO com CO.

\section{AGRADECIMENTOS}

Ao Departamento de Engenharia Química da Universidade Federal de São Carlos e ao CNPq.

\section{REFERÊNCIAS}

1. Tejuca, L.G.: Fierro, J. L. G.; Properties and Applications of PerovskiteType Oxides, Marcel Dekker: New York, 1993.

2. Ortiz, J. I. G.; Rivas, B.; Fonseca, R. L.; Velasco, J. R. G.; Appl. Catal., A 2004, 26, 147.

3. Machida, M.; Ochiai, K.; Ito, K.; Ikeue, K.; Catal. Today 2006, 117, 584.

4. Urasaki K.; Tokunaga, K.; Sekine, Y.; Matsukata, M.; Kikuchi, E.; Catal. Commun. 2008, 9, 600.

5. Sdiri, N.; Bejar, M.; Hussein, M.; Mazen, S.; Dhahri, E.; J. Magn. Magn. Mater. 2007, 7, 703.

6. Tanaka, H.; Mizuno, N.; Misono, M.; Appl. Catal., A 2003, 244, 371.
7. Liu, Y.; Zheng, H.; Liu, J.; Zhang, T.; Chem. Eng. J. 2002, 89, 213.

8. Garcia, J. S.; Dissertação de Mestrado, Universidade de São Paulo, Brasil, 2003.

9. Rougier, A.; Soiron, S.; Haihal, I.; Aymard, L.; Taouk, B.; Tarascon, J.; Powder Technol. 2002, 128, 139.

10. Joint Committee on Powder Diffraction Standards (JCPDS): International Center of Diffraction Data, Pensilvânia, 1995.

11. Dupeyrat, C. B.; Valderrama, G.; Meneses, A.; Martinez, F.; Barraul, J.; Tatibouet, J. M.; Appl. Catal. A 2003, 143, 248.

12. Wang, W.; Zhang, H.; Lin, G.; Xiong, Z.; Appl. Catal., B 2000, 24 , 219.

13. Lee, Y.; Lago, R.; Fierro, J. L.; Cortés, V.; Sapina, F.; Martinez, E.; Appl. Catal., A 2001, 207, 17.

14. Aristova, I. M.; Sedykh, V. D.; Shekhtman, V.; Kulakov, V. I.; Mater. Lett. 2008, 62, 1036.

15. Rojas, M.; Fierro, J. L.; Tejuca, L. G.; Bell, A. T.; J. Catal. 1990, 124, 41.

16. Patcas, F.; Buciuman, F. C.; Zsako, J.; Thermochim. Acta 2000, 360, 71.

17. Niu, J.; Deng, J.; Liu, W.; Wang, G.; Zi, X.; Catal. Today 2007, 126, 409.

183 Tanabe, E. Y.; Dissertação de Mestrado, Universidade de São Paulo, Brasil, 2006.

19. Buciuman, F. C.; Patcas, F.; Zsakó, J.; J. Therm. Anal. Calorim. 2000, 61,819 . 